OPEN ACCESS

Check for updates

\section{Health outcomes of young children born to mothers who received 2009 pandemic H1N1 influenza vaccination during pregnancy: retrospective cohort study}

\author{
Laura K Walsh, ${ }^{1,2}$ Jessy Donelle, ${ }^{3}$ Linda Dodds, ${ }^{4}$ Steven Hawken, ${ }^{2,3,5}$ Kumanan Wilson, $, 3,5$ \\ Eric I Benchimol, 2,3,6 Pranesh Chakraborty, ${ }^{2,6}$ Astrid Guttmann, 3,7,8 Jeffrey C Kwong, 3,7,9,10 \\ Noni E MacDonald, ${ }^{4}$ Justin R Ortiz, ${ }^{11}$ Ann E Sprague, ${ }^{1,2,6}$ Karina A Top, ${ }^{4}$ Mark C Walker, ${ }^{1,2,5}$ \\ Shi Wu Wen, ${ }^{2,5}$ Deshayne B Fell ${ }^{2,3,6}$
}

${ }^{1}$ Better Outcomes Registry \& Network, Ottawa, ON, Canada ${ }^{2}$ University of Ottawa, Ottawa, ON, Canada

${ }^{3}$ ICES, Toronto, ON, Canada ${ }^{4}$ Dalhousie University, Halifax, NS, Canada

${ }^{5}$ Ottawa Hospital Research Institute, Ottawa, ON, Canada

${ }^{6}$ Children's Hospital of Eastern Ontario (CHEO) Research

Institute, Ottawa, ON, Canada

${ }^{7}$ Dalla Lana School of Public Health, University of Toronto, Toronto, ON, Canada

${ }^{8}$ Hospital for Sick Children, Toronto, ON, Canada

${ }^{9}$ Public Health Ontario, Toronto, ON, Canada

${ }^{10}$ Department of Family and Community Medicine, University of Toronto, Toronto, ON, Canada

${ }^{11}$ University of Maryland School of Medicine, Baltimore, MD, USA

Correspondence to: D Fell dfell@cheo.on.ca

(ORCID 0000-0002-5548-3228) Additional material is published online only. To view please visit the journal online.

Cite this as: BMJ 2019;366:14151 http://dx.doi.org/10.1136/bmj.14151

Accepted: 21 May 2019

\section{ABSTRACT}

\section{OBJECTIVE}

To determine whether any association exists between exposure to 2009 pandemic H1N1 (pH1N1) influenza vaccination during pregnancy and negative health outcomes in early childhood.

\section{DESIGN}

Retrospective cohort study.

SETTING

Population based birth registry linked with health administrative databases in the province of Ontario, Canada.

\section{PARTICIPANTS}

All live births from November 2009 through October 2010 ( $n=104249$ ) were included, and children were followed until 5 years of age to ascertain study outcomes.

\section{MAIN OUTCOME MEASURES}

Rates of immune related (infectious diseases, asthma), non-immune related (neoplasms, sensory disorders), and non-specific morbidity outcomes (urgent or inpatient health services use, pediatric complex chronic conditions) were evaluated from birth to 5 years of age; under- 5 childhood mortality was also assessed. Propensity score weighting was used to adjust hazard ratios, incidence rate ratios, and risk ratios for potential confounding.

\section{RESULTS}

Of 104249 live births, 31295 (30\%) were exposed to $\mathrm{pH} 1 \mathrm{~N} 1$ influenza vaccination in utero. No significant associations were found with upper or lower respiratory infections, otitis media, any infectious diseases, neoplasms, sensory disorders, urgent and inpatient health services use, pediatric complex

\section{WHAT IS ALREADY KNOWN ON THIS TOPIC}

According to recent research, concern about safety is commonly cited as a reason for low uptake of influenza vaccine among pregnant women

Lack of information on long term health outcomes in children exposed to influenza vaccination in utero is a potential barrier to achieving higher vaccine uptake

\section{WHAT THIS STUDY ADDS}

No association was observed between exposure to 2009 pandemic H1N1 influenza vaccine during pregnancy and most five year pediatric health outcomes These results support the safety profile of 2009 pandemic H1N1 influenza vaccination during pregnancy chronic conditions, or mortality. A weak association was observed between prenatal $\mathrm{pH} 1 \mathrm{~N} 1$ vaccination and increased risk of asthma (adjusted hazard ratio $1.05,95 \%$ confidence interval 1.02 to 1.09 ) and decreased rates of gastrointestinal infections (adjusted incidence rate ratio $0.94,0.91$ to 0.98 ). These results were unchanged in sensitivity analyses accounting for any potential differential healthcare seeking behavior or access between exposure groups.

\section{CONCLUSIONS}

No associations were observed between exposure to $\mathrm{pH} 1 \mathrm{~N} 1$ influenza vaccine during pregnancy and most five year pediatric health outcomes. Residual confounding may explain the small associations observed with increased asthma and reduced gastrointestinal infections. These outcomes should be assessed in future studies.

\section{Introduction}

Pregnant women are considered to be at high risk for serious illness due to influenza related mortality and morbidity documented during influenza pandemics and seasonal epidemics. ${ }^{1-13}$ In the United States and Canada, policies advising all pregnant women to be immunized against influenza have been in place for many years. ${ }^{14-18}$ Similar policies now exist in other countries, ${ }^{19} 20$ many of which were implemented in response to the $2009 \mathrm{H} 1 \mathrm{~N} 1$ influenza pandemic. ${ }^{20}$ In addition to directly protecting pregnant women, vaccine derived maternal antibodies cross the placenta and confer passive immunity to infants during the first months of life. ${ }^{21-26}$ As infants under 6 months of age have the highest burden of morbidity and mortality associated with pediatric influenza, ${ }^{27-29}$ but influenza vaccines are not licensed for use in this group, ${ }^{30}$ immunization during pregnancy is an important strategy for protecting young infants from influenza infection. $^{31}$

Despite the strong evidence of benefit to mothers and newborns, uptake of influenza vaccination during pregnancy has been low, even when recommended and funded. ${ }^{32}{ }^{33}$ Concern about safety is a commonly cited reason for pregnant women not being immunized, ${ }^{34-37}$ and this can also affect healthcare providers' willingness to recommend influenza vaccination to their pregnant patients. ${ }^{37}$ Although substantial evidence now supports the safety of maternal influenza immunization with respect to birth outcomes (for example, preterm birth, congenital anomalies), ${ }^{38-43}$ 
few studies have assessed pediatric health outcomes beyond the first six months of life. ${ }^{44-49}$ The lack of information on longer term health outcomes in children following influenza vaccination during pregnancy may be a potential barrier to achieving higher uptake and has been identified as an evidence gap for maternal immunization policy globally. ${ }^{50} 51$ In this study, we evaluated the relation between 2009 pandemic H1N1 (pH1N1) influenza vaccination during pregnancy and pediatric health outcomes in the first five years of life.

\section{Methods}

Study design, data sources, and study population

We did a population based retrospective cohort study of infants born to residents of Ontario, Canada from 2 November 2009 through 31 October 2010. We used the Better Outcomes Registry \& Network (BORN) Ontario birth registry (https://www.bornontario.ca/ en/about-born/) to identify the study population. This province-wide registry contains maternal-newborn records for all hospital births of at least $500 \mathrm{~g}$ or at least 20 weeks of gestational age. In addition to routine sociodemographic and clinical data, information on receipt of the monovalent 2009 pH1N1 influenza vaccine during pregnancy was captured as part of enhanced surveillance during the 2009 influenza pandemic. We linked this one year birth cohort with health administrative databases to ascertain pediatric health outcomes over a five year follow-up period. All databases were linked using unique encoded identifiers and analyzed at ICES (https://www.ices.on.ca/). The administrative databases included the Canadian Institute for Health Information's Discharge Abstract Database (hospital admissions) and the National Ambulatory Care Reporting System (emergency department visits), each containing clinical diagnoses made during healthcare encounters, coded using the Canadian adaptation of the international classification of diseases, 10th revision (ICD-10-CA) system, as well as the Ontario Asthma dataset, which is derived from the administrative databases. We additionally linked with the Ontario Cancer Registry to identify cases of pediatric cancer and with the Registered Persons Database to derive follow-up time for each child and to ascertain childhood mortality. Further description of the data sources and linkage methodology is provided in supplementary methods 1 .

We excluded infants whose birth registry record could not be linked to the administrative databases, those born to women who were not continuously eligible to receive healthcare in Ontario during pregnancy, those with records with data quality problems (for example, duplicates, invalid date of birth), and those not eligible for publicly funded provincial healthcare at birth. We also excluded infants whose records were missing information on 2009 pH1N1 influenza vaccination during pregnancy or who died on their date of birth.

\section{Exposure and outcome measurement}

The exposure of interest was receipt of the monovalent 2009 pH1N1 influenza vaccine during pregnancy, ascertained from database specific codes in the birth registry. We classified infants born to mothers with documented 2009 pH1N1 influenza immunization during pregnancy as exposed to vaccine and those whose mothers were not immunized against 2009 pH1N1 influenza during pregnancy as unexposed. The pandemic influenza vaccination campaign in Ontario started on 26 October 2009. In Canada, two pandemic vaccines were produced (both by GlaxoSmithKline Biologicals)-an unadjuvanted pH1N1 influenza vaccine, specifically intended for use in pregnant women, and an AS03 adjuvanted product (Arepanrix) produced for the general population. The second one was not contraindicated in pregnancy if the unadjuvanted product was unavailable and the risk of influenza was deemed to be high. ${ }^{5253}$

A consensus list of standardized case definitions has been developed for monitoring obstetric and neonatal outcomes following immunization during pregnancy. ${ }^{54-56}$ In the absence of such guidance for later pediatric health outcomes and the limited research on this topic, we pre-specified three groups of childhood morbidity outcomes for our evaluation. Firstly, we were primarily interested in immune related outcomes (infectious and atopic diseases), as the developing fetal immune system is thought to be sensitive to influences such as maternal immunization. ${ }^{57-59}$ To assess safety, we included two non-immune related morbidity outcomes that have been used in other safety studies among pregnant women (neoplasms, sensory disorders) ${ }^{60}$ and two non-specific morbidity outcomes (urgent and inpatient health services use, pediatric complex chronic conditions). Where possible, we used clinical registries and standardized validated algorithms to identify outcomes. When no relevant registry or established algorithm was available, we measured outcomes by using diagnostic codes from emergency department visits and hospital admissions, but not from outpatient primary care visits, in an effort to limit the analysis to cases with better measurement in the available databases, as well as more serious clinical implications. Subsequent to developing our original study protocol, we included childhood mortality up to the age of 5 years as an additional outcome.

Infectious outcomes included upper and lower respiratory tract infections, gastrointestinal infections, otitis media, and a composite of these four categories of infections. Sensory disorders included vision and hearing loss combined. We searched for ICD-10CA diagnostic codes for each of these outcomes in primary or secondary diagnostic code field positions of the hospital admission and emergency department databases. Diagnoses of asthma were ascertained from the Ontario Asthma dataset, which uses a validated algorithm (sensitivity 89\%, specificity $72 \%$ ) to identify cases of asthma from health administrative databases. ${ }^{61}$ Children in our cohort who were in the Ontario Asthma dataset but coded with a diagnosis of asthma before 6 months of age were not classified as asthma cases unless a diagnostic code for asthma 
could be found in the hospital admission or emergency department databases later during follow-up. ${ }^{62}$ Confirmed diagnoses of pediatric cancer came from the Ontario Cancer Registry. We modified an existing algorithm to identify children with a complex chronic condition (expected to last more than 12 months and need specialty care, likely including hospital admission in a tertiary care center). ${ }^{63}$ We ascertained child mortality from the Registered Persons Database. See supplementary tables A and B for a list of diagnostic codes used to identify all morbidity outcomes.

\section{Statistical analyses}

We described the characteristics of the study population by using frequencies for categorical variables and medians (interquartile ranges) for continuous variables. We used standardized differences to assess the balance of baseline covariates between the two exposure groups, with an absolute standardized difference below 0.10 considered indicative of a well balanced covariate. ${ }^{64}$

We used weights derived from propensity scores to adjust for confounding in our study. We developed a logistic regression model to calculate a propensity score for each infant, representing the predicted probability of 2009 pH1N1 influenza immunization during pregnancy. Before running the propensity score models, we used multiple imputation to correct for missing values for covariates $-6.9 \%$ of records had missing information for one or more covariates that we intended to include in the propensity score; the percentage of missing data for any one of the individual variables was less than $1 \%$ for most (rural residence, public health unit region, parity, fifths of neighborhood income) and was highest for maternal smoking during pregnancy (3.9\%). We included the following pre-selected covariates from the birth registry in the propensity score models, which were developed using each of the 10 multiple imputation datasets: maternal age, parity, maternal smoking, season of conception, antenatal care provider, maternal preexisting medical comorbidity, obstetric complications, use of antenatal steroids, multifetal gestation, fifth of neighborhood income, rural residence, and public health unit region. Subsequently, we developed inverse probability of treatment weights (IPTWs), whereby the value for each vaccine exposed infant was the inverse of the propensity score and the value for each unexposed infant was the inverse of 1 minus the propensity score. ${ }^{65}$ To stabilize any extreme weights, we standardized to the entire study population by multiplying by the marginal propensity score.

Follow-up began on the date of birth and continued until the child either became ineligible for healthcare in Ontario (owing to emigration or death) or reached 5 years of age. However, for time-to-event outcomes (asthma, neoplasms, sensory disorders, mortality), the end of follow-up was the event date for those experiencing the outcome. We used Cox proportional hazards models (asthma, neoplasms, sensory disorders, mortality), negative binomial models (infectious disease outcomes, urgent and inpatient health services use), and log binomial models (pediatric complex chronic conditions) to estimate unadjusted and adjusted hazard ratios, incidence rate ratios, and risk ratios, respectively, with $95 \%$ confidence intervals. To generate adjusted results, we ran weighted models for each outcome by using the stabilized IPTWs generated from each of the 10 multiple imputation datasets. We then statistically combined the resulting parameters and standard errors to produce a single adjusted point estimate and 95\% confidence interval. For the Cox models, we found the proportional hazards assumption for the exposure variable to be fulfilled on the basis of examination of Schoenfeld residual plots and Wald tests for interaction between exposure status and time.

In sensitivity analyses, we examined those outcomes for which the $95 \%$ confidence interval around the adjusted point estimated excluded the null value. Firstly, we restricted the study population to infants with at least two well baby visits or primary immunization visits in the first year of life to ensure that the child was accessing the healthcare system (see supplementary methods 2). We also repeated our main analyses with additional adjustment for the number of maternal outpatient visits within six months before the index pregnancy and the number of non-obstetric hospital admissions within two years before the start of the index pregnancy to account for any differences in healthcare seeking or access. For the asthma outcome, we also further adjusted for maternal asthma status. We recalculated the confidence intervals after applying a Bonferroni correction to account for multiple comparisons ( $n=10$ pre-specified morbidity outcomes) and explored a negative control outcome (rate of all cause injuries) to assess possible rival explanations. ${ }^{66}$ Finally, we did several additional analyses to characterize the effect of excluding records with missing information on $\mathrm{pH} 1 \mathrm{~N} 1$ vaccination. We used SAS version 9.4 for all analyses.

\section{Patient involvement}

This research was done without patient involvement. Patients were not consulted to develop the research question, nor were they involved in identifying the study design or outcomes. We did not invite any patients to participate in the interpretation of results, nor in the writing or editing of this document. There are no plans to directly involve patients in the dissemination of these research findings.

\section{Results}

Of 135807 live births in the Ontario birth registry that were initially eligible, 4714 (3.5\%) could not be linked with administrative databases, and we excluded $13687(10 \%)$ for administrative reasons. Of the 117406 remaining infants, we excluded $77(<1 \%)$ who died on their date of birth, as well as $13080(11 \%)$ who were missing information on $2009 \mathrm{pH} 1 \mathrm{~N} 1$ vaccination during pregnancy, leaving 104249 live births in our final study population (fig 1). A total of 31295 (30\%) 
children were born to women vaccinated against 2009 pH1N1 influenza during pregnancy. In the unweighted study population, vaccinated women were more likely to be over 30 years of age and live in the highest fifth of neighborhood income. After weighting with stabilized IPTWs, we found all measured baseline covariates to be well balanced (absolute standardized difference $<0.1$ ) indicating an improvement in the comparability of children born to women who were and were not vaccinated with $2009 \mathrm{pH} 1 \mathrm{~N} 1$ influenza vaccine during pregnancy (table 1).

The median length of follow-up of pH1N1 influenza vaccine exposed and unexposed infants was five years. A total of 14459 children (14\%) received a diagnosis of asthma during follow-up, at a median age of 1.8 years. The proportion of children diagnosed as having asthma was highest among those born to mothers with a preexisting medical comorbidity, particularly asthma, and those born preterm (see supplementary table C). Among infectious study outcomes, 34\% ( $n=35$ 441) of children had at least one upper respiratory tract infection during follow-up (see supplementary table C). Less than $1 \%$ of children were diagnosed as having a sensory disorder (152 (0.15\%); see supplementary table D), neoplasm (145 (0.14\%); supplementary table D), or pediatric complex chronic condition (444 (0.87\%); supplementary table E).

We did not observe any significant associations between prenatal exposure to $2009 \mathrm{pH} 1 \mathrm{~N} 1$ influenza vaccination and upper or lower respiratory infections, otitis media, all infections, neoplasms, sensory disorders, rates of urgent and inpatient health services use, pediatric complex chronic conditions, or under-5 mortality. We found a weak, but statistically significant, increased association with asthma (adjusted hazard ratio 1.05 , 95\% confidence interval 1.02 to 1.09), as well as an inverse association with gastrointestinal infections (adjusted incidence rate ratio $0.94,0.91$ to 0.98), even after accounting for potential confounding in weighted analyses (table 2 ).

We subjected our findings for asthma and gastrointestinal infections to several sensitivity analyses (see supplementary table F). Firstly, to ensure contact with the healthcare system during the first year of life, we restricted the analysis to children with at least two well baby or routine immunization visits in their first year; however, the positive association with asthma and inverse association with gastrointestinal infections were both unchanged. Additional adjustment for maternal propensity to access healthcare before pregnancy also had a negligible effect on these point estimates. When we used a Bonferroni correction to account for multiplicity, neither the association with asthma (adjusted hazard ratio 1.05, 1.00 to 1.11) nor the association with gastrointestinal infections (adjusted incidence rate ratio $0.94,0.88$ to 1.00 ) remained statistically significant. We observed a small, statistically significant increase in rates of all cause injuries (our negative control outcome) in infants exposed to $2009 \mathrm{pH} 1 \mathrm{~N} 1$ influenza vaccine compared with unexposed infants (adjusted incidence rate ratio
$1.03,1.01$ to 1.05 ), and this association persisted in all sensitivity analyses. Approximately $11 \%$ of records were missing information on $\mathrm{pH} 1 \mathrm{~N} 1$ vaccination during pregnancy and were excluded from our analyses. When we examined the distribution of baseline study variables in infants missing exposure information ( $n=13080$ infants) and those included in our analyses ( $n=104249)$, we found them to be generally comparable. Any differences in characteristics across these two groups (that is, with a standardized difference $>0.1$ ) were largely due to missing data patterns in other variables (supplementary table G). Differences in study outcomes between these two groups were very small in magnitude and not clinically informative (see supplementary table $\mathrm{H}$ ), and sensitivity analyses to assess the potential effect of the missing data did not alter our findings or interpretation (supplementary table I).

\section{Discussion}

In this large, population based study of 104249 children, 31295 of whom were born to women vaccinated against 2009 pH1N1 influenza during pregnancy, we found no associations between exposure to the $2009 \mathrm{pH} 1 \mathrm{~N} 1$ influenza vaccine in utero and pediatric upper respiratory tract infections, lower respiratory tract infections, otitis media, all infections, neoplasms, sensory disorders, rates of urgent and inpatient health services use, pediatric complex chronic conditions, or mortality up to 5 years of age.

Although we observed an increased association between maternal 2009 pH1N1 influenza vaccination and pediatric asthma, the small magnitude of the association, which was no longer statistically significant after we accounted for multiplicity, along with evidence of a small correlation with our negative control outcome, implies that these results should be interpreted cautiously. We also observed a significant inverse association between 2009 pH1N1 influenza vaccination during pregnancy and pediatric gastrointestinal infections that persisted in all sensitivity analyses. Although we used propensity score methods to adjust for differences between vaccine exposed and unexposed infants, this association could still be due to residual confounding, possibly by an increased likelihood of vaccinated mothers to ensure rotavirus vaccination in their children. Although the publicly funded rotavirus immunization program in Ontario was not operational during our study period, the vaccine was available by prescription for purchase. ${ }^{67}$ We attempted to account for rotavirus immunization through a sensitivity analysis limited to infants accessing regular well baby and primary immunization visits during the first year; however, no rotavirus specific vaccine codes were available to allow for specific adjustment in our models.

\section{Strengths and weaknesses of study}

The main strength of our study was the availability of a population based birth registry with detailed clinical information on pregnancy and birth, receipt of the 


\begin{tabular}{|c|c|c|c|c|c|c|c|}
\hline \multirow[b]{2}{*}{ Characteristic } & \multirow{2}{*}{$\begin{array}{l}\text { Whole population } \\
\text { (unweighted) } \\
\text { ( } n=104249)\end{array}$} & \multicolumn{3}{|c|}{$\begin{array}{l}2009 \text { pH1N1 influenza vaccination during pregnancy } \\
\text { (unweighted) }\end{array}$} & \multicolumn{3}{|c|}{$\begin{array}{l}2009 \mathrm{pH} 1 \mathrm{~N} 1 \text { influenza vaccination } \\
\text { during pregnancy } \\
\text { (\%, IPTW weighted*) }\end{array}$} \\
\hline & & Yes $(n=31295)$ & No $(n=72954)$ & $\begin{array}{l}\text { Standardized } \\
\text { differencet }\end{array}$ & Yes & No & $\begin{array}{l}\text { Standardized } \\
\text { differencet }\end{array}$ \\
\hline \multicolumn{8}{|l|}{ Maternal age, years: } \\
\hline$<20$ & $3582(3.4)$ & $735(2.4)$ & $2847(3.9)$ & 0.09 & 3.5 & 3.5 & 0.002 \\
\hline $20-24$ & $13183(12.7)$ & $2846(9.1)$ & $10337(14.2)$ & 0.16 & 11.5 & 13.1 & 0.048 \\
\hline $25-29$ & $29023(27.8)$ & $7980(25.5)$ & $21043(28.8)$ & 0.08 & 27.8 & 27.8 & 0.001 \\
\hline $30-34$ & $35583(34.1)$ & $11902(38.0)$ & $23681(32.5)$ & 0.12 & 36.3 & 32.9 & 0.072 \\
\hline$\geq 35$ & $22878(22.0)$ & $7832(25.0)$ & $15046(20.6)$ & 0.11 & 20.9 & 22.7 & 0.042 \\
\hline \multicolumn{8}{|l|}{ Parity: } \\
\hline 0 (nulliparous) & $44634(42.8)$ & $13100(41.9)$ & $31534(43.2)$ & 0.03 & 42.4 & 42.8 & 0.008 \\
\hline$\geq 1$ (multiparous) & $59369(57.0)$ & $18107(57.9)$ & $41262(56.6)$ & 0.03 & 57.4 & 57.0 & 0.007 \\
\hline Missing & $246(0.2)$ & $88(0.3)$ & $158(0.2)$ & 0.01 & 0.3 & 0.2 & 0.012 \\
\hline \multicolumn{8}{|c|}{ Smoking during pregnancy: } \\
\hline No & $88604(85.0)$ & $27075(86.5)$ & $61529(84.3)$ & 0.06 & 84.5 & 85.0 & 0.014 \\
\hline Yes & 11594 (11.1) & $2850(9.1)$ & $8744(12.0)$ & 0.09 & 11.7 & 11.2 & 0.014 \\
\hline Missing & $4051(3.9)$ & $1370(4.4)$ & $2681(3.7)$ & 0.04 & 3.9 & 3.8 & 0.002 \\
\hline \multicolumn{8}{|c|}{ Pre-existing maternal medical conditionsł: } \\
\hline No & $86917(83.4)$ & $25630(81.9)$ & $61287(84.0)$ & 0.06 & 83.3 & 83.4 & 0.003 \\
\hline Yes & $17332(16.6)$ & $5665(18.1)$ & $11667(16.0)$ & 0.06 & 16.7 & 16.6 & 0.003 \\
\hline Asthma§ & $14771(14.2)$ & $4786(15.3)$ & $9985(13.7)$ & 0.05 & 14.2 & 14.2 & 0.001 \\
\hline $\begin{array}{l}\text { Chronic } \\
\text { hypertension§ }\end{array}$ & $847(0.8)$ & $324(1.0)$ & $523(0.7)$ & 0.03 & 0.8 & 0.8 & 0.002 \\
\hline Diabetes§ & $1823(1.8)$ & $582(1.9)$ & $1241(1.7)$ & 0.01 & 1.8 & 1.8 & 0.003 \\
\hline Heart disease§ & $479(0.5)$ & $179(0.6)$ & $300(0.4)$ & 0.02 & 0.5 & 0.5 & 0.003 \\
\hline \multicolumn{8}{|c|}{ Obstetric complications $\uparrow:$} \\
\hline No & 93118 (89.3) & 27827 (88.9) & $65291(89.5)$ & 0.02 & 89.1 & 89.4 & 0.009 \\
\hline Yes & $11131(10.7)$ & $3468(11.1)$ & $7663(10.5)$ & 0.02 & 10.9 & 10.6 & 0.009 \\
\hline \multicolumn{8}{|l|}{ Multiple birth: } \\
\hline No & $100579(96.5)$ & $30032(96.0)$ & $70547(96.7)$ & 0.04 & 96.5 & 96.5 & 0.000 \\
\hline Yes & $3670(3.5)$ & $1263(4.0)$ & $2407(3.3)$ & 0.04 & 3.5 & 3.5 & 0.000 \\
\hline \multicolumn{8}{|c|}{ Fifth of neighborhood median family income: } \\
\hline 1 (lowest) & $23035(22.1)$ & $5535(17.7)$ & $17500(24.0)$ & 0.16 & 22.3 & 22.1 & 0.004 \\
\hline 2 & $20434(19.6)$ & $5631(18.0)$ & $14803(20.3)$ & 0.06 & 19.7 & 19.6 & 0.001 \\
\hline 3 & $21196(20.3)$ & $6358(20.3)$ & $14838(20.3)$ & 0.00 & 20.1 & 20.3 & 0.003 \\
\hline 4 & $22421(21.5)$ & $7281(23.3)$ & $15140(20.8)$ & 0.06 & 21.5 & 21.5 & 0.000 \\
\hline 5 (highest) & $16714(16.0)$ & $6377(20.4)$ & $10337(14.2)$ & 0.16 & 16.0 & 16.0 & 0.001 \\
\hline Missing & $449(0.4)$ & $113(0.4)$ & $336(0.5)$ & 0.02 & 0.4 & 0.5 & 0.007 \\
\hline \multicolumn{8}{|l|}{ Rural residence: } \\
\hline Yes & $10488(10.1)$ & $3433(11.0)$ & $7055(9.7)$ & 0.04 & 10.5 & 10.1 & 0.012 \\
\hline No/missing & 93761 (89.9) & $27862(89.0)$ & $65899(90.3)$ & 0.04 & 89.5 & 89.9 & 0.021 \\
\hline \multicolumn{8}{|c|}{ Gestational age at birth in weeks: } \\
\hline Term ( $\geq 37$ weeks) & $96315(92.4)$ & $28955(92.5)$ & $67360(92.3)$ & 0.01 & 92.8 & 92.3 & 0.012 \\
\hline \multicolumn{8}{|c|}{ Preterm } \\
\hline$<28$ & $302(0.3)$ & $81(0.3)$ & $221(0.3)$ & 0.01 & 0.3 & 0.3 & 0.010 \\
\hline $28-31$ & $697(0.7)$ & $189(0.6)$ & $508(0.7)$ & 0.01 & 0.6 & 0.7 & 0.011 \\
\hline $32-33$ & $945(0.9)$ & $285(0.9)$ & $660(0.9)$ & 0.00 & 0.8 & 0.9 & 0.008 \\
\hline 34 & $985(0.9)$ & $285(0.9)$ & $700(1.0)$ & 0.01 & 0.9 & 1.0 & 0.008 \\
\hline 35 & $1692(1.6)$ & $478(1.5)$ & $1214(1.7)$ & 0.01 & 1.4 & 1.7 & 0.020 \\
\hline 36 & $3313(3.2)$ & $1022(3.3)$ & 2291 (3.1) & 0.01 & 3.2 & 3.2 & 0.001 \\
\hline \multicolumn{8}{|l|}{ Infant sex: } \\
\hline Male & $53667(51.5)$ & $16127(51.5)$ & $37540(51.5)$ & 0.002 & 51.5 & 51.4 & 0.002 \\
\hline Female & $50582(48.5)$ & $15168(48.5)$ & $35414(48.5)$ & 0.002 & 48.5 & 48.6 & 0.002 \\
\hline \multicolumn{8}{|c|}{$\begin{array}{l}\text { IPTW=inverse probability of treatment weight. } \\
\text { *Stabilized inverse probability of treatment weighting. } \\
\text { †Absolute standardized difference, whereby value }>0.10 \text { indicates imbalance between } 2009 \text { pH1N1 influenza vaccine exposed and unexposed } \\
\text { participants. } \\
\text { †Asthma, chronic hypertension, diabetes, or heart disease. } \\
\text { \$Sum of each individual condition does not equal number of women with any condition, as categories were not mutually exclusive. } \\
\text { १Pregnancy induced hypertension, pre-eclampsia, eclampsia, gestational diabetes, placenta previa, or placental abruption. }\end{array}$} \\
\hline
\end{tabular}

2009 pH1N1 influenza vaccine during pregnancy, ${ }^{44}$ and linkage to health administrative databases permitting follow-up for health outcomes up to 5 years of age. We cannot dismiss the possibility of residual confounding of our findings due to an inability to include unmeasured or unknown confounders in the propensity scores. ${ }^{68}$ However, this method has been shown to reduce bias more effectively than conventional multivariable adjustment methods in studies using large administrative databases, ${ }^{69}$ 


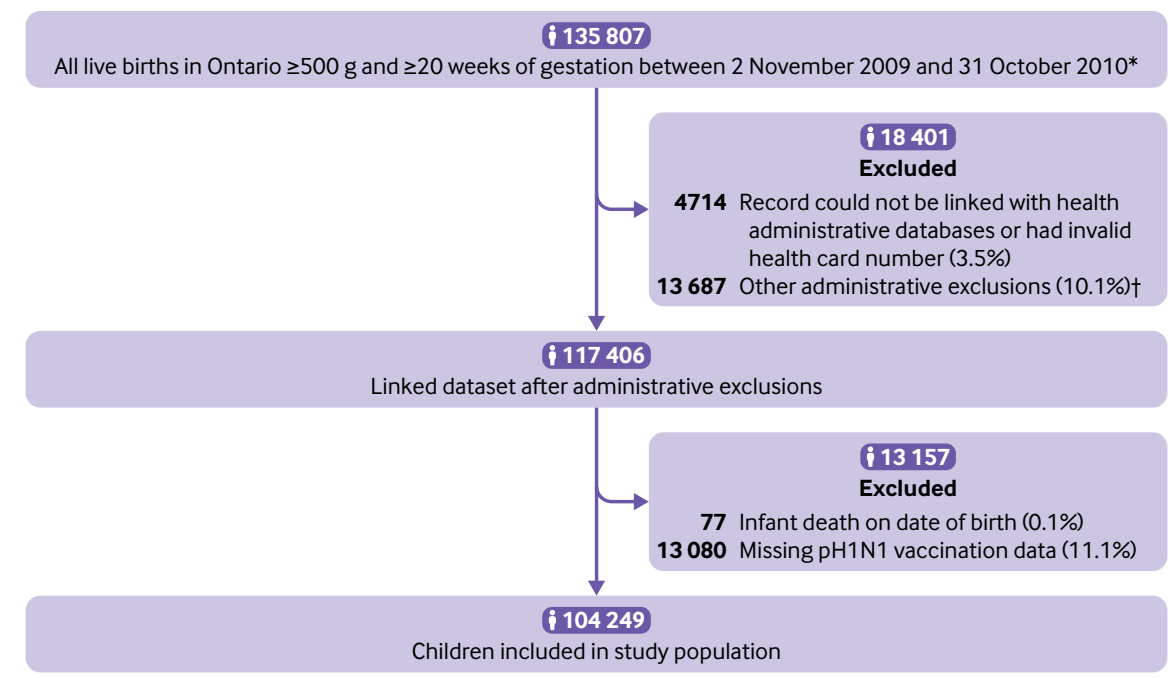

Fig 1 | Flow diagram of study. *Information on maternal 2009 pandemic H1N1 influenza vaccination was collected between 2 November 2009 and 31 October 2010. Invalid birth date $(n=21)$, duplicate record $(n=31)$, invalid linkage $(n=209)$, women who were not continuously eligible to receive healthcare in Ontario during pregnancy or infant not eligible at birth for provincial healthcare $(n=13426)$

including studies of influenza vaccine. ${ }^{70}$ With the exception of neoplasms, for which we used a clinical registry, we recognize the possibility of measurement error of other study outcomes. We used a validated algorithm to identify cases of pediatric asthma ${ }^{61}$; however, clinically confirmed diagnoses of asthma would have been preferable. Although we believe that any misclassification of asthma status would likely have been non-differential according to exposure, vaccinated women may have been more likely to access healthcare for their infants, and this could have biased our results away from the null. Nevertheless, our sensitivity analyses designed to account for maternal propensity to access healthcare did not change any of the results. In sensitivity analyses, we did not find any evidence to suggest that the exclusion of records with missing information on $\mathrm{pH} 1 \mathrm{~N} 1$ influenza vaccination during pregnancy would have introduced significant bias. In addition to similar frequencies of baseline characteristics and risk of study outcomes among records with and without complete information on pH1N1 vaccination, repeating our analyses within subgroups of the study population with lower percentages of records with missing $\mathrm{pH} 1 \mathrm{~N} 1$ influenza

\begin{tabular}{|c|c|c|c|c|c|c|}
\hline \multirow[b]{2}{*}{ Outcome } & \multicolumn{2}{|c|}{$\begin{array}{l}2009 \mathrm{pH} 1 \mathrm{~N} 1 \text { influenza vaccination } \\
\text { during pregnancy }\end{array}$} & \multicolumn{2}{|c|}{$\begin{array}{l}\text { No } 2009 \text { pH1N1 influenza vaccination } \\
\text { during pregnancy }\end{array}$} & \multirow[b]{2}{*}{$\begin{array}{l}\text { Crude estimate } \\
(95 \% \mathrm{Cl})\end{array}$} & \multirow[b]{2}{*}{$\begin{array}{l}\text { Adjusted estimate } \\
(95 \% \mathrm{Cl})^{\star}\end{array}$} \\
\hline & No of events & $\begin{array}{l}\text { Incidence rate }(95 \% \mathrm{Cl}) \\
\text { per } 1000 \text { person years }\end{array}$ & No of events & $\begin{array}{l}\text { Incidence rate }(95 \% \mathrm{Cl}) \\
\text { per } 1000 \text { person years }\end{array}$ & & \\
\hline \multicolumn{7}{|l|}{ Immune related morbidity outcomes: } \\
\hline \multicolumn{7}{|l|}{ Atopic disease: } \\
\hline Asthmat & 4359 & 30.7 (29.9 to 31.6) & 10100 & $30.6(30.0$ to 31.1$)$ & $1.00(0.97$ to 1.04$)$ & $1.05(1.02$ to 1.09$)$ \\
\hline \multicolumn{7}{|l|}{ Infectious diseases: } \\
\hline Upper respiratory tract infectionsł & 20132 & $130.6(128.2$ to 132.9$)$ & 46677 & $130.0(128.4$ to 131.5$)$ & 1.01 (0.98 to 1.03$)$ & $1.01(0.98$ to 1.03$)$ \\
\hline Lower respiratory tract infections & 8102 & $52.5(51.4$ to 53.7$)$ & 19335 & $53.8(53.1$ to 54.6$)$ & $0.98(0.94$ to 1.01$)$ & $0.99(0.95$ to 1.03$)$ \\
\hline Gastrointestinal infections $\ddagger$ & 6138 & 39.8 (38.9 to 40.7$)$ & 15320 & 42.7 (42.1 to 43.3$)$ & $0.93(0.90$ to 0.97$)$ & 0.94 (0.91 to 0.98) \\
\hline Otitis mediał & 10129 & 65.7 (64.3 to 67.1$)$ & 22732 & $63.3(62.4$ to 64.2$)$ & 1.04 (1.00 to 1.07$)$ & $1.03(1.00$ to 1.06$)$ \\
\hline All infections $\ddagger$ & 44501 & $288.6(284.1$ to 293.2$)$ & 104064 & $289.7(286.8$ to 292.7$)$ & $1.00(0.97$ to 1.04$)$ & $1.01(0.98$ to 1.03$)$ \\
\hline \multicolumn{7}{|l|}{ Non-immune related morbidity outcomes: } \\
\hline Neoplasmst & 48 & $0.30(0.29$ to 0.31$)$ & 97 & 0.27 (0.26 to 0.28$)$ & 1.12 (0.79 to 1.59$)$ & $1.12(0.79$ to 1.59$)$ \\
\hline Sensory disorderst & 42 & $0.27(0.26$ to 0.28$)$ & 110 & $0.30(0.29$ to 0.30$)$ & 0.89 (0.62 to 1.27$)$ & $0.94(0.67$ to 1.33$)$ \\
\hline \multicolumn{7}{|l|}{ Non-specific morbidity outcomes: } \\
\hline Urgent and inpatient health services use $\ddagger$ & 104043 & $674.7(666.5$ to 683.1$)$ & 246292 & $685.7(680.2$ to 691.2$)$ & $0.98(0.97$ to 1.00$)$ & $0.99(0.98$ to 1.01$)$ \\
\hline Pediatric complex chronic condition§ & 134 & $0.88(0.85$ to 0.91$)$ & 310 & $0.87(0.85$ to 0.89$)$ & $1.01(0.82$ to 1.23$)$ & $0.98(0.80$ to 1.20$)$ \\
\hline 5 year mortality $\uparrow$ & 77 & $0.50(0.47$ to 0.53$)$ & 220 & $0.61(0.59$ to 0.63$)$ & $0.83(0.63$ to 1.06$)$ & 0.83 (0.64 to 1.08$)$ \\
\hline \multicolumn{7}{|c|}{$\begin{array}{l}\text { *Adjusted using stabilized inverse probability of treatment weights. } \\
\text { tNumber of events represents total unweighted number of children diagnosed as having outcome. Point estimates shown are hazards ratios generated using Cox proportion hazards regression } \\
\text { model. } \\
\text { fNumber of events represents total unweighted number of occurrences for each outcome. Point estimates shown are incidence rate ratios generated using negative binomial regression model. } \\
\text { \$Number of events represents total unweighted number of children diagnosed as having two or more of composite chronic conditions. Original indicator also includes categories of conditions } \\
\text { related to prematurity and congenital or genetic defects. These have been excluded from modified indicator. Point estimates shown are risk ratios generated using log binomial regression model, } \\
\text { restricted to infants with full } 5 \text { years of follow-up ( } n=101611 \text { ). } \\
\text { qAnalysis based on number of deaths up to age of } 5 \text { years, excluding those that occurred on date of birth. }\end{array}$} \\
\hline
\end{tabular}


vaccination information yielded results that were consistent with our primary analyses. Although an unadjuvanted $\mathrm{pH} 1 \mathrm{~N} 1$ influenza vaccine was produced specifically for pregnant women in Canada during the pandemic, ${ }^{5253}$ some women probably received the AS03 adjuvanted vaccine used in the general population. We were unable to assess the products separately, as no distinction was made in the birth registry. Finally, despite the large size of our study, we were likely still underpowered to rule out small associations for the rarest outcomes.

\section{Comparison with other studies}

Despite longstanding recommendations for influenza vaccination during pregnancy, ${ }^{14-20}$ coverage has been suboptimal (for example, $<20 \%$ in Canada, ${ }^{32} 54 \%$ in the United States ${ }^{33}$ ), possibly owing to ongoing concern about safety of vaccination during pregnancy. ${ }^{343571}$ To date, most studies of influenza immunization during pregnancy that include follow-up of infant outcomes beyond the early neonatal period have limited their assessment to the first six months and focused exclusively on influenza outcomes (for example, laboratory confirmed influenza, hospital admission for influenza). ${ }^{2122} 252672-78$ To our knowledge, only six studies have assessed health outcomes in children beyond 6 months of age. ${ }^{44-49}$ Two followed infants from birth to 1 year and compared outcomes between 2009 $\mathrm{pH} 1 \mathrm{~N} 1$ vaccinated mothers and unvaccinated mothers. No differences were observed in developmental scores or infection related physician visits in a small cohort of Dutch infants born to women immunized with MF59 adjuvanted $\mathrm{pH} 1 \mathrm{~N} 1$ vaccine, ${ }^{45}$ nor in rates of influenza infection or overall health services use (emergency department visits and hospital admissions) in a large study from Canada, conducted in the same population as our study. ${ }^{48}$ The remaining four studies assessed outcomes beyond the first year of life. The first, from 1977, did not identify any association between maternal seasonal influenza immunization and neurologic outcomes, although important study limitations existed, including a small sample size. ${ }^{47}$ More recently, Hviid and colleagues did a retrospective cohort study using Danish registry data from the 2009 H1N1 pandemic time period. Among 61359 infants ( 6311 born to women vaccinated with an AS03 adjuvanted pH1N1 influenza vaccine), no increased risk of various childhood morbidities was observed across a five year follow-up period (for example, infectious diseases and neurologic, autoimmune, or behavioral conditions). ${ }^{44}$ The Danish study found no association between maternal 2009 pH1N1 influenza vaccination and pediatric asthma (first trimester risk ratio $1.50,0.99$ to 2.29 ; second/third trimester $1.02,0.89$ to 1.16$)$. Interestingly, a significant inverse association between 2009 pH1N1 influenza vaccination and pediatric gastrointestinal infections was also observed in the Danish cohort (risk ratio $0.84,0.74$ to 0.94$).^{44}$ Similarly to our assessment of under-5 mortality, a Swedish study assessed mortality in offspring from 7 days of life to 4.6 years following
pH1N1 influenza vaccination during pregnancy with an AS03 adjuvanted vaccine, finding no association (hazard ratio $0.97,0.69$ to 1.36$).{ }^{49}$ Finally, no overall increase in the risk of autism spectrum disorder was observed following influenza vaccination during pregnancy (seasonal trivalent influenza vaccine or unadjuvanted pH1N1 influenza vaccine) in a California based cohort study of infants born between 2000 and $2010 .{ }^{46}$

\section{Conclusions}

Overall, our findings indicate that 2009 pH1N1 influenza vaccination in pregnancy was not associated with negative five year health outcomes in children, which is reassuring and consistent with a similar recent study from Denmark. Although we observed a small, but statistically significant, increase in pediatric asthma and a reduction in gastrointestinal infections, we are not aware of any biologic mechanisms to explain these findings. Future studies in different settings and with different influenza vaccine formulations will be important for developing the evidence base on longer term pediatric outcomes following influenza vaccination during pregnancy.

We thank Melanie Varin for her assistance with formatting the manuscript and Sarah Spruin for her assistance with analytical revisions. We are grateful to Better Outcomes Registry \& Network (BORN) Ontario, Ottawa, Canada, and ICES for providing data access.

This study is based in part on data provided by BORN, part of the Children's Hospital of Eastern Ontario. The interpretation and conclusions contained herein do not necessarily represent those of BORN Ontario. Parts of this material are based on data and/ or information compiled and provided by the Canadian Institute for Health Information (CIHI). However, the analyses, conclusions, opinions, and statements expressed in the material are those of the authors and not necessarily those of $\mathrm{ClHI}$. Parts of this material are based on data and information provided by Cancer Care Ontario (CCO). The opinions, results, view, and conclusions reported in this paper are those of the authors and do not necessarily reflect those of CCO. No endorsement by CCO is intended or should be inferred.

Contributors: All authors contributed to the study concept and design, as well as the acquisition, analysis, or interpretation of data. LKW and JD did the statistical analysis. DBF and LKW drafted the manuscript. All authors critically reviewed the manuscript for important intellectual content. DBF obtained the funding and supervised the study. The corresponding author attests that all listed authors meet authorship criteria and that no others meeting the criteria have been omitted. DBF had full access to all the data in the study and takes responsibility for the integrity of the data and the accuracy of the data analysis. LKW and DBF are the guarantors.

Funding: This research was supported by an operating grant from the Canadian Institutes of Health Research (A01-151541) and by ICES, which is funded by an annual grant from the Ontario Ministry of Health and Long term Care (MOHLTC). EIB is supported by a new investigator award from the Canadian Institutes of Health Research, Canadian Association of Gastroenterology, and Crohn's and Colitis Canada. He is also supported by the Career Enhancement Program of the Canadian Child Health Clinician Scientist Program. JCK is supported by a clinician-scientist award from the Department of Family and Community Medicine, University of Toronto. The funders of this research were not involved in the study design, data analysis, or manuscript preparation or publication decisions. The opinions, results, and conclusions reported in this article are those of the authors and are independent from the funding sources. No endorsement by ICES or the Ontario MOHLTC is intended or should be inferred.

Competing interests: All authors have completed and submitted the ICMJE uniform disclosure form at www.icmje.org/coi_disclosure.pdf (available on request from the corresponding author) and declare: support for the work as described above; KAT has received grants from GlaxoSmithKline and personal fees and non-financial support from 
Pfizer, both of which were unrelated to the submitted work; no other relationships or activities that could appear to have influenced the submitted work.

Ethical approval: This study received approval from the Ottawa Health Science Network Research Ethics Board (protocol No 20170431- 01H), the Children's Hospital of Eastern Ontario Research Ethics Board (protocol No 17/04PE), and the ICES Privacy Office (protocol No 20180901137 000).

Data sharing: The dataset from this study is held securely in coded form at ICES. Although data sharing agreements prohibit ICES from making the dataset publicly available, access may be granted to those who meet pre-specified criteria for confidential access, available at www.ices.on.ca/DAS. The full dataset creation plan and underlying analytic code are available from the authors on request, understanding that the computer programs may rely on coding templates or macros that are unique to ICES and, therefore, either are inaccessible or may need modification.

Transparency: The manuscript's guarantors affirm that the manuscript is an honest, accurate, and transparent account of the study being reported; that no important aspects of the study have been omitted; and that any discrepancies from the study as originally planned (and, if relevant, registered) have been explained.

This is an Open Access article distributed in accordance with the Creative Commons Attribution Non Commercial (CC BY-NC 4.0) license, which permits others to distribute, remix, adapt, build upon this work non-commercially, and license their derivative works on different terms, provided the original work is properly cited and the use is noncommercial. See: http://creativecommons.org/licenses/by-nc/4.0/.

1 Rothberg MB, Haessler SD, Brown RB. Complications of viral influenza. Am J Med 2008;121:258-64. doi:10.1016/j. amjmed.2007.10.040

2 Centers for Disease Control. Influenza. In: Atkinson W, Wolfe S, Hamborsky J, eds. Epidemiology and Prevention of VaccinePreventable Diseases. 12th ed. Public Health Foundation, 2012.

3 Steinhoff MC. Epidemiology and prevention of influenza. In: Nelson K, Masters Williams C, eds. Infectious Disease Epidemiology. Theory and Practice. 2nd ed. Jones and Bartlett Publishers, 2007: 577-600.

4 Mertz D, Kim TH, Johnstone J, et al. Populations at risk for severe or complicated influenza illness: systematic review and meta-analysis. BMJ 2013;347:f5061. doi:10.1136/bmj.f5061

5 Siston AM, Rasmussen SA, Honein MA, et al. Pandemic H1N1 Influenza in Pregnancy Working Group. Pandemic 2009 influenza $\mathrm{A}(\mathrm{H} 1 \mathrm{~N} 1)$ virus illness among pregnant women in the United States. IAMA 2010;303:1517-25. doi:10.1001/jama.2010.479

6 Harris J. Influenza occurring in pregnant women. J Am Med Assoc 1919;72:978-80. doi:10.1001/jama.1919.02610140008002.

7 Freeman DW, Barno A. Deaths from Asian influenza associated with pregnancy. Am / Obstet Gynecol 1959;78:1172-5. doi:10.1016/0002-9378(59)90570-8

8 Low DE, McGeer A. Pandemic (H1N1) 2009: assessing the response. CMA/ 2010;182:1874-8. doi:10.1503/cmaj.100900

9 Jamieson DJ, Honein MA, Rasmussen SA, et al. Novel Influenza A (H1N1) Pregnancy Working Group. H1N1 2009 influenza virus infection during pregnancy in the USA. Lancet 2009;374:451-8. doi:10.1016/S0140-6736(09)61304-0

10 Kumar A, Zarychanski R, Pinto R, et al. Canadian Critical Care Trials Group H1N1 Collaborative. Critically ill patients with 2009 influenza A(H1N1) infection in Canada. JAMA 2009;302:1872-9. doi:10.1001/jama.2009.1496

11 Dodds L, McNeil SA, Fell DB, et al. Impact of influenza exposure on rates of hospital admissions and physician visits because of respiratory illness among pregnant women. CMA/ 2007;176:463-8. doi:10.1503/cmaj.061435

12 Schanzer DL, Langley JM, Tam TWS. Influenza-attributed hospitalization rates among pregnant women in Canada 1994-2000. J Obstet Gynaecol Can 2007;29:622-9. doi:10.1016/S17012163(16)32559-2

13 Neuzil KM, Reed GW, Mitchel EF, Simonsen L, Griffin MR. Impact of influenza on acute cardiopulmonary hospitalizations in pregnant women. Am J Epidemiol 1998;148:1094-102. doi:10.1093/ oxfordjournals.aje.a009587

14 Grohskopf LA, Sokolow LZ, Broder KR, et al. Prevention and Control of Seasonal Influenza with Vaccines: Recommendations of the Advisory Committee on Immunization Practices - United States, 2017-18 Influenza Season. MMWR Recomm Rep 2017;66:1-20. doi:10.15585/mmwr.rr6602a1

15 American College of Obstetricians and Gynecologists. ACOG Committee Opinion No. 741: Maternal Immunization. Obstet Gynecol 2018;131:e214-7. doi:10.1097/ AOG.0000000000002662
16 National Advisory Committee on Immunization (NACI). Canadian Immunization Guide Chapter on Influenza and Statement on Seasonal Influenza Vaccine for 2018-2019. 2018. https://www. canada.ca/en/public-health/services/publications/healthy-living/ canadian-immunization-guide-statement-seasonal-influenzavaccine-2018-2019.html\#pre.

17 Castillo E, Poliquin V. No. 357-Immunization in Pregnancy. J Obstet Gynaecol Can 2018;40:478-89. doi:10.1016/j.jogc.2017.11.010

18 Skowronski DM, De Serres G. Is routine influenza immunization warranted in early pregnancy?Vaccine 2009;27:4754-70. doi:10.1016/j.vaccine.2009.03.079

19 Mak TK, Mangtani P, Leese J, Watson JM, Pfeifer D. Influenza vaccination in pregnancy: current evidence and selected national policies. Lancet Infect Dis 2008;8:44-52. doi:10.1016/S14733099(07)70311-0

20 Ortiz IR, Perut M, Dumolard L, et al. A global review of national influenza immunization policies: Analysis of the 2014 WHO/UNICEF Joint Reporting Form on immunization. Vaccine 2016;34:5400-5. doi:10.1016/j.vaccine.2016.07.045

21 Madhi SA, Cutland CL, Kuwanda L, et al. Maternal Flu Trial (Matflu) Team. Influenza vaccination of pregnant women and protection of their infants. N EnglJ Med 2014;371:918-31. doi:10.1056/ NEJMoa1401480

22 Tapia MD, Sow SO, Tamboura B, et al. Maternal immunisation with trivalent inactivated influenza vaccine for prevention of influenza in infants in Mali: a prospective, active-controlled, observer-blind, randomised phase 4 trial. Lancet Infect Dis 2016;16:1026-35. doi:10.1016/S1473-3099(16)30054-8

23 Tsatsaris V, Capitant C, Schmitz T, et al. Inserm C09-33 PREFLUVAC (Immunogenicity and Safety of an Inactivated Nonadjuvanted A[H1N1v] Influenza Vaccine in Pregnant Women) Study Group. Maternal immune response and neonatal seroprotection from a single dose of a monovalent nonadjuvanted 2009 influenza A(H1N1) vaccine: a single-group trial. Ann Intern Med 2011;155:733-41. doi:10.7326/0003-4819-155-11-201112060-00005

24 Zuccotti G, Pogliani L, Pariani E, Amendola A, Zanetti A. Transplacental antibody transfer following maternal immunization with a pandemic 2009 influenza A(H1N1) MF59-adjuvanted vaccine. IAMA 2010;304:2360-1. doi:10.1001/jama.2010.1729

25 Zaman K, Roy E, Arifeen SE, et al. Effectiveness of maternal influenza immunization in mothers and infants. N Engl J Med 2008;359:155564. doi:10.1056/NEJMoa0708630

26 Steinhoff MC, Katz J, Englund JA, et al. Year-round influenza immunisation during pregnancy in Nepal: a phase 4, randomised, placebo-controlled trial. Lancet Infect Dis 2017;17:981-9. doi:10.1016/S1473-3099(17)30252-9

27 Nair H, Brooks WA, Katz M, et al. Global burden of respiratory infections due to seasonal influenza in young children: a systematic review and meta-analysis. Lancet 2011;378:1917-30. doi:10.1016/ S0140-6736(11)61051-9

28 Poehling KA, Edwards KM, Griffin MR, et al. The burden of influenza in young children, 2004-2009. Pediatrics 2013;131:207-16. doi:10.1542/peds.2012-1255

29 Bhat N, Wright JG, Broder KR, et al. Influenza Special Investigations Team. Influenza-associated deaths among children in the United States, 2003-2004. N Engl I Med 2005;353:2559-67. doi:10.1056/ NEJMoa051721

30 Fiore AE, Uyeki TM, Broder K, et al. Centers for Disease Control and Prevention (CDC). Prevention and control of influenza with vaccines: recommendations of the Advisory Committee on Immunization Practices (ACIP), 2010. MMWR Recomm Rep 2010;59(RR-8):1-62.

31 Omer SB. Maternal Immunization. N Engl I Med 2017;376:2497. doi:10.1056/NEJMra1509044

32 Legge A, Dodds L, MacDonald NE, Scott J, McNeil S. Rates and determinants of seasonal influenza vaccination in pregnancy and association with neonatal outcomes. CMAI 2014;186:E157-64. doi:10.1503/cmaj.130499

33 Ding $\mathrm{H}$, Black CL, Ball S, et al. Influenza vaccination coverage among pregnant women - United States, 2016-17 influenza season. MMWR Morb Mortal Wkly Rep 2017;66:1016-22. doi:10.15585/mmwr. mm6638a2

34 Yuen CY, Tarrant M. Determinants of uptake of influenza vaccination among pregnant women - a systematic review. Vaccine 2014;32:4602-13. doi:10.1016/j.vaccine.2014.06.067

35 Chamberlain AT, Seib K, Ault KA, et al. Factors associated with intention to receive influenza and tetanus, diphtheria, and acellular pertussis (Tdap) vaccines during pregnancy: a focus on vaccine hesitancy and perceptions of disease severity and vaccine safety. PLoS Curr 2015;7:ecurrents.outbreaks. d37b61bceebae5a7a06d40a301cfa819. doi:10.1371/currents. outbreaks.d37b61bceebae5a7a06d40a301cfa819

36 Wilson RJ, Paterson P, Jarrett C, Larson HJ. Understanding factors influencing vaccination acceptance during pregnancy globally: A literature review. Vaccine 2015;33:6420-9. doi:10.1016/j. vaccine.2015.08.046 
37 MacDougall DM, Halperin SA. Improving rates of maternal immunization: Challenges and opportunities. Hum Vaccin Immunother 2016:12:857-65. doi:10.1080/21645515.2015.1101524

38 Moro PL, Broder K, Zheteyeva Y, et al. Adverse events following administration to pregnant women of influenza A (H1N1) 2009 monovalent vaccine reported to the Vaccine Adverse Event Reporting System. Am J Obstet Gynecol 2011;205:473. doi:10.1016/j. ajog.2011.06.047

39 Moro PL, Tepper NK, Grohskopf LA, Vellozzi C, Broder K. Safety of seasonal influenza and influenza A (H1N1) 2009 monovalent vaccines in pregnancy. Expert Rev Vaccines 2012;11:911-21. doi:10.1586/erv.12.72

40 Keller-Stanislawski B, Englund JA, Kang G, et al. Safety of immunization during pregnancy: a review of the evidence of selected inactivated and live attenuated vaccines. Vaccine 2014;32:7057-64. doi:10.1016/j. vaccine.2014.09.052

41 Fell DB, Platt RW, Lanes A, et al. Fetal death and preterm birth associated with maternal influenza vaccination: systematic review. BJOG 2015:122:17-26. doi:10.1111/1471-0528.12977

42 McMillan M, Porritt K, Kralik D, Costi L, Marshall H. Influenza vaccination during pregnancy: a systematic review of fetal death, spontaneous abortion, and congenital malformation safety outcomes. Vaccine 2015:33:2108-17. doi:10.1016/ivaccine.2015.02.068

43 Bratton KN, Wardle MT, Orenstein WA, Omer SB. Maternal influenza immunization and birth outcomes of stillbirth and spontaneous abortion: a systematic review and meta-analysis. Clin Infect Dis 2015;60:e11-9. doi:10.1093/cid/ciu915

44 Hviid A, Svanström H, Mølgaard-Nielsen D, Lambach P. Association between pandemic influenza $\mathrm{A}(\mathrm{H} 1 \mathrm{~N} 1)$ vaccination in pregnancy and early childhood morbidity in offspring. JAMA Pediatr 2017;171:23948. doi:10.1001/jamapediatrics.2016.4023

45 van der Maas N, Dijs-Elsinga J, Kemmeren J, van Lier A, Knol M, de Melker H. Safety of vaccination against influenza A (H1N1) during pregnancy in the Netherlands: results on pregnancy outcomes and infant's health: cross-sectional linkage study. BJOG 2016;123:70917. doi:10.1111/1471-0528.13329

46 Zerbo O, Qian Y, Yoshida C, Fireman BH, Klein NP, Croen LA Association between influenza infection and vaccination during pregnancy and risk of autism spectrum disorder. JAMA Pediatr 2017:171:e163609. doi:10.1001/jamapediatrics.2016.3609

47 Heinonen O, Slone D, Shapiro S. Immunizing agents. In: Kaufman D, ed. Birth Defects and Drugs in Pregnancy. Publishing Sciences Group, 1977: 314-21.

48 Fell DB, Wilson K, Ducharme R, et al. Infant respiratory outcomes associated with prenatal exposure to maternal 2009 A/H1N1 influenza vaccination. PLoS One 2016;11:e0160342. doi:10.1371/ journal.pone.0160342

49 Ludvigsson JF, Ström P, Lundholm C, et al. Maternal vaccination against H1N1 influenza and offspring mortality: population based cohort study and sibling design. BMJ 2015;351:h5585. doi:10.1136/bmj.h5585

50 Ortiz JR, Neuzil KM, Ahonkhai VI, et al. Translating vaccine policy into action: a report from the Bill \& Melinda Gates Foundation Consultation on the prevention of maternal and early infant influenza in resource-limited settings. Vaccine 2012;30:7134-40. doi:10.1016/i.vaccine.2012.09.034

51 Fell DB, Bhutta ZA, Hutcheon JA, et al. Report of the WHO technical consultation on the effect of maternal influenza and influenza vaccination on the developing fetus: Montreal, Canada, September 30-October 1, 2015. Vaccine 2017;35:2279-87. doi:10.1016/j. vaccine. 2017.03 .056

52 Harris T, Wong K, Stanford L, Fediurek J, Crowcroft N, Deeks S. Did narcolepsy occur following administration of ASO3-adjuvanted $\mathrm{A}(\mathrm{H} 1 \mathrm{~N} 1)$ pandemic vaccine in Ontario, Canada? A review of postmarketing safety surveillance data. Euro Surveill 2014;19:20900. doi:10.2807/1560-7917.ES2014.19.36.20900

53 Skowronski DM, Janjua NZ, De Serres G, et al. Effectiveness of ASO3 adjuvanted pandemic H1N1 vaccine: case-control evaluation based on sentinel surveillance system in Canada, autumn 2009. BM/ 2011;342:c7297. doi:10.1136/bmi.c7297

54 Kochhar S, Bauwens J, Bonhoeffer J, et al, GAIA Project Participants. Electronic address: http://www.gaia-consortium.net/. Safety assessment of immunization in pregnancy. Vaccine 2017;35(48 Pt A):6469-71. doi:10.1016/j.vaccine.2017.09.033

55 Bonhoeffer J, Kochhar S, Hirschfeld SGAIA project participants. Global alignment of immunization safety assessment in pregnancy - The GAIA project. Vaccine 2016;34:5993-7. doi:10.1016/j. vaccine.2016.07.006

56 Munoz FM, Eckert LO, Katz MA, et al. Key terms for the assessment of the safety of vaccines in pregnancy: Results of a global consultative process to initiate harmonization of adverse event definitions. Vaccine 2015;33:6441-52. doi:10.1016/j. vaccine.2015.07.112

57 MacGillivray DM, Kollmann TR. The role of environmental factors in modulating immune responses in early life.
Front Immunol 2014;5:434. doi:10.3389/ fimmu.2014.00434

58 Chen T, Liu H-X, Yan H-Y, Wu D-M, Ping J. Developmental origins of inflammatory and immune diseases. Mol Hum Reprod 2016;22:858 65. doi:10.1093/molehr/gaw036

59 Barker DJP, Thornburg KL. The obstetric origins of health for a lifetime. Clin Obstet Gynecol 2013;56:511-9. doi:10.1097/ GRF.0b013e31829cb9ca

60 Ray JG, Vermeulen MJ, Bharatha A, Montanera WJ, Park AL. Association between MRI exposure during pregnancy and fetal and childhood outcomes. JAMA 2016;316:952-61. doi:10.1001/ jama.2016.12126

61 Gershon AS, Wang C, Guan J, Vasilevska-Ristovska J, Cicutto L, To T. Identifying patients with physician-diagnosed asthma in health administrative databases. Can Respir / 2009;16:183-8. doi:10.1155/2009/963098

62 Radhakrishnan DK, Dell SD, Guttmann A, Shariff SZ, Liu K, To T. Trends in the age of diagnosis of childhood asthma. J Allergy Clin Immunol 2014:134:1057-62.e5. doi:10.1016/j.jaci.2014.05.012

63 Feudtner C, Feinstein JA, Zhong W, Hall M, Dai D. Pediatric complex chronic conditions classification system version 2 : updated for ICD-10 and complex medical technology dependence and transplantation. BMC Pediatr 2014;14:199. doi:10.1186/14712431-14-199

64 Austin PC. Using the standardized difference to compare the prevalence of a binary variable between two groups in observational research. Commun Stat Simul Comput 2009;38:1228-34. doi:10.1080/03610910902859574.

65 Cole SR, Hernán MA. Constructing inverse probability weights for marginal structural models. Am J Epidemiol 2008;168:656-64. doi:10.1093/aje/kwn164

66 Lipsitch M, Tchetgen Tchetgen E, Cohen T. Negative controls: a tool for detecting confounding and bias in observational studies. Epidemiology 2010;21:383-8. doi:10.1097/ EDE.0b013e3181d61eeb

67 Wilson SE, Chung H, Schwartz KL, et al. Rotavirus vaccine coverage and factors associated with uptake using linked data: Ontario, Canada. PLoS One 2018;13:e0192809. doi:10.1371/journal. pone.0192809

68 Brookhart MA, Wyss R, Layton JB, Stürmer T. Propensity score methods for confounding control in nonexperimental research. Circ Cardiovasc Qual Outcomes 2013;6:604-11. doi:10.1161/ CIRCOUTCOMES 113.000359

69 Austin PC, Stuart EA. Moving towards best practice when using inverse probability of treatment weighting (IPTW) using the propensity score to estimate causal treatment effects in observational studies. Stat Med 2015;34:3661-79. doi:10.1002/ $\operatorname{sim} .6607$

70 Schneeweiss S, Rassen JA, Glynn RJ, Avorn J, Mogun H, Brookhart MA. High-dimensional propensity score adjustment in studies of treatment effects using health care claims data. Epidemiology 2009;20:512-22. doi:10.1097/EDE.0b013e3181a663cc

71 Top KA, Arkell C, Scott H, et al. Effect of package insert language on health-care providers' perceptions of influenza vaccination safety during pregnancy. Lancet Glob Health 2016;4:e690-1. doi:10.1016/ S2214-109X(16)30182-6

72 Munoz FM, Greisinger AJ, Wehmanen OA, et al. Safety of influenza vaccination during pregnancy. Am J Obstet Gynecol 2005;192:1098 106. doi:10.1016/j.ajog.2004.12.019

73 Benowitz I, Esposito DB, Gracey KD, Shapiro ED, Vázquez M Influenza vaccine given to pregnant women reduces hospitalization due to influenza in their infants. Clin Infect Dis 2010;51:1355-61. doi:10.1086/657309

74 Eick AA, Uyeki TM, Klimov A, et al. Maternal influenza vaccination and effect on influenza virus infection in young infants. Arch Pediatr Adolesc Med 2011:165:104-11. doi:10.1001/ archpediatrics.2010.192

75 Poehling KA, Szilagyi PG, Staat MA, et al, New Vaccine Surveillance Network. Impact of maternal immunization on influenza hospitalizations in infants. Am J Obstet Gynecol 2011;204(Suppl 1):S141-8. doi:10.1016/j.ajog.2011.02.042

76 Black SB, Shinefield HR, France EK, Fireman BH, Platt ST, Shay DVaccine Safety Datalink Workgroup. Effectiveness of influenza vaccine during pregnancy in preventing hospitalizations and outpatient visits for respiratory illness in pregnant women and their infants. Am J Perinatol 2004;21:333-9. doi:10.1055/s-2004-831888

77 Shakib JH, Korgenski K, Presson AP, et al. Influenza in infants born to women vaccinated during pregnancy. Pediatrics 2016;137:e20152360. doi:10.1542/peds.2015-2360.

78 Sukumaran L, McCarthy NL, Kharbanda EO, et al. Infant hospitalizations and mortality after maternal vaccination. Pediatrics 2018;141:e20173310. doi:10.1542/peds.2017-3310

Supplementary materials 